\title{
PESQUISA BIBLIOGRÁFICA SOBRE OS ESTUDOS \\ CIENTÍFICOS RELACIONADOS COM O BITCOIN E A \\ BLOCKCHAIN
}

\author{
BIBLIOGRAPHIC RESEARCH ON SCIENTIFIC STUDIES \\ RELATED TO BITCOIN AND BLOCKCHAIN
}

\section{UNA PESQUISA BIBLIOGRÁFICA SOBRE CÓMO LA CIENCIA INVESTIGA EL BITCOIN Y LA TECNOLOGÍA BLOCKCHAIN}

\author{
Juan Arturo Castañeda-Ayarza \\ Professor-pesquisador na Pontifícia Universidade \\ Católica de Campinas, Brasil \\ Doutor em Planejamento de Sistemas Energéticos \\ pela Universidade Estadual de Campinas, Brasil \\ juan.fem@gmail.com
}

\section{Cíntia Neves}

Graduada em Administração de Empresas pela Pontifícia Universidade Católica de Campinas, Brasil

nevescintia@me.com

\section{André Frazão Teixeira}

Docente da Faculdade La Salle de Manaus, Brasil Doutor em Planejamento de Sistemas Energéticos pela Universidade Estadual de Campinas, Brasil andre.teixeira@outlook.com

\author{
Contextus \\ ISSNe 2178-9258 \\ Organização: Comitê Científico Interinstitucional \\ Editor-Chefe: Diego de Queiroz Machado \\ Editora associada: Alane Siqueira Rocha \\ Avaliação: double blind review pelo SEER/OJS \\ Recebido em 25/08/2019 \\ Aceito em 04/10/2019 \\ Versão final aceita em 21/10/2019 \\ http://dx.doi.org/10.19094/contextus.v17i3.41986
}

\begin{abstract}
RESUMO
No desenvolvimento das tecnologias de informação e internet, as moedas digitais representam uma nova dinâmica tecnológica e monetária. Em 2008, surgiu o Bitcoin, uma moeda virtual em forma de código de computador que se caracteriza por estar livre de vínculos com autoridades monetárias e por não possuir nacionalidade. $\mathrm{O}$ crescente interesse no Bitcoin também é encontrado na ciência. Assim, nosso objetivo foi mapear o conhecimento científico desenvolvido acerca do Bitcoin e da tecnologia Blockchain. Através da pesquisa bibliográfica em duas bases de dados, Science Direct e Scielo, mapearam-se, entre 2008 e 2018, 432 publicações, das quais $80 \%$ aconteceram entre 2016 e 2018, evidenciando-se o acelerado crescimento da produção científica. Os principais assuntos pesquisados estão relacionados com o mercado de Bitcoin, a segurança e eficiência da tecnologia Blockchain, o mercado ilegal, além das potenciais aplicações em áreas como energia, saúde, economia compartilhada, Big Data e Internet das Coisas.
\end{abstract}

Palavras-chave: criptomoeda; blockchain; criptomercado; bitcoin; pesquisa bibliográfica.

\begin{abstract}
Digital currencies represent a new technological and monetary dynamics in the development of information technologies and internet. Bitcoin, a virtual currency that emerged in 2008 as a computer code, is characterized by being free of ties to monetary authorities and by not having nationality. The growing interest in Bitcoin is also found in science. Thus, our objective was to map the scientific knowledge about Bitcoin and Blockchain technology. Through bibliographic research in two databases, Science Direct and Scielo, 432 publications were mapped between 2008 and 2018. Out of these, 80\% happened between 2016 and 2018, highlighting the rapid growth of scientific production. The main research topics are related to the Bitcoin market, the safety and efficiency of Blockchain technology, the illegal market, as well as the potential applications in areas such as energy, health, shared economy, Big Data and Internet of Things.
\end{abstract}

Keywords: Cryptocurrency; Blockchain; Crypto Market; Bitcoin; Bibliographic Research. 


\section{RESUMEN}

En las tecnologías de información y la Internet, las monedas digitales representan un nuevo nivel de dinámica tecnológica y monetaria. En 2008 surgió el Bitcoin, un concepto de moneda en forma de código de computador, que se caracteriza por estar libre de vínculos con autoridades monetarias y por no tener nacionalidad. Su creciente interés también ha llamado la atención de la ciencia. Nuestro objetivo fue mapear el conocimiento científico desarrollado acerca del Bitcoin y de la tecnología Blockchain. Se realizó una pesquisa bibliográfica en dos bases de datos, Science Direct y Scielo, mapeándose las publicaciones entre 2008 y 2018. Se identificaron 432 publicaciones, siendo 80\% entre 2016 y 2018, evidenciándose acelerado crecimiento de la producción científica. Los principales asuntos investigados están relacionados al mercado de Bitcoin, la seguridad y eficiencia de la tecnología Blockchain, el mercado ilegal, además de las potenciales aplicaciones en áreas como energía, salud, economía compartida, Big Data e Internet de las Cosas.

Palabras clave: criptomoneda; blockchain; criptomercado; bitcoin; estudio bibliográfico.

\section{INTRODUÇÃO}

No desenvolvimento das tecnologias de informação e, consequentemente, da internet, as moedas digitais representam um novo patamar na dinâmica tecnológica e, principalmente, no âmbito monetário. Em paralelo à moeda oficial, esta nova forma de realizar transações iniciou um modo singular de trocas financeiras, que operam exclusivamente por meio virtual e sem a necessidade do controle das instituições financeiras, como os bancos centrais (CALVETTE, 2015).

É válido voltar alguns anos na história para entender que uma crise financeira instalada nos EUA - onde em 2008, o banco de investimentos Lehman Brothers foi à falência - deixou em evidência a fragilidade da situação da moeda e dos agentes que intervêm na administração e funcionamento da mesma. Foi a partir desse cenário que, no mesmo ano e se aproveitando de ideias já existentes, adveio uma publicação sob autoria do autonomeado Satoshi Nakamoto, chamada "Bitcoin: A Peer-to-Peer Electronic Cash System", propondo um novo conceito de moeda na forma de código de computador, incluindo uma nova forma de operação, livre de vínculos com autoridades monetárias e sem ligação com nenhuma nacionalidade (SILVEIRA, 2015).

A repercussão dessa publicação, juntamente com a necessidade de um desenvolvimento progressivo do sistema monetário e com o auxílio da evolução dos meios de comunicação e a digitalização do sistema bancário financeiro, permitiram que essa proposta de inovação e outras ideias de modernização na era digital fossem possíveis, surgindo assim um novo meio de pagamento, não regulamentado e sem vínculo com as autoridades financeiras, possibilitando a abertura de um mercado de moedas eletrônicas (BAUR; HONG; LEE, 2018). 
Este mercado está atualmente em crescimento e divide opiniões. Aqueles que veem futuro nas moedas digitais e no sistema de verificação e garantia de trocas utilizado por elas, estão entusiasmados com as várias possibilidades de transações, verificadas e publicamente registradas, através de operações fáceis, uma vez que tudo isto é feito automaticamente, sem burocracias e, por isso, dão uma visão otimista para o futuro desse tipo de moeda, seja como meio de troca ou forma de investimento (NEW SCIENTIST, 2013). Entretanto, há aqueles que acreditam que, no longo prazo, esse processo entrará em colapso, uma vez que as moedas digitais são como commodities, finitas e por isso limitadas (DYHRBERG, 2016).

O Bitcoin é totalmente descentralizado e depende de um protocolo sofisticado, que se utiliza da criptografia para gerenciar o suprimento das transações e tenta, assim, evitar ações que possam prejudicar ou colocar em risco o sistema. Todas as transações são registradas e armazenadas digitalmente, através de uma tecnologia que compartilha dados contábeis, conhecida como Blockchain (BALCILAR, 2017).

A Blockchain é uma tecnologia que permite contabilizar os registros de todas as operações realizadas com a moeda Bitcoin, fornecendo rapidez e segurança para os usuários (DWYER, 2015; PINZÓN; ROCHA, 2016).

Para Eyal e Sirer (2014), a segurança da Blockchain reside no protocolo que é distribuído na cadeia de transações executadas pelos participantes, conhecidos como miners ou mineradores. No entanto, esse protocolo tem mostrado vulnerabilidades, através de ataques realizados pelos mesmos mineradores. Em adição, Pinto (2015) destaca como vantagens do Bitcoin e seu sistema Blockchain a transparência das transações e o menor custo das operações, mas ressalta os riscos da falta de regulamentação, esta ausência também verificada por Silva e Monteiro (2018).

A partir da contextualização relatada, entende-se que o hodierno campo do Bitcoin, assim como de outras moedas digitais, tem motivado o interesse de especialistas financeiros, que defendem ou detratam o seu futuro. Ao mesmo tempo, acadêmicos de diversas áreas do conhecimento têm mostrado interesse no Bitcoin e na tecnologia Blockchain, contribuindo com publicações em importantes revistas científicas desde 2008.

Sendo assim, identifica-se como oportunidade de pesquisa a importância de mapear e mostrar o desenvolvimento do conhecimento científico em relação às criptomoedas. Por isso, o objetivo deste trabalho é elaborar uma pesquisa bibliográfica sobre a contribuição que a ciência vem apresentando sobre o estudo e a aplicabilidade teórica e prática do Bitcoin e da 
tecnologia Blockchain.

Este artigo segue a seguinte estrutura: a primeira seção, apresentada até este momento, está dedicada à contextualização, problematização e formulação do objetivo principal da pesquisa. A segunda seção, Referencial Teórico, apresenta os principais conceitos relacionados com os assuntos da pesquisa. A seção 3 descreve o método de pesquisa bibliográfica utilizado. A seção 4 apresenta, analisa e discute os resultados e, finalmente, a seção 5 é dedicada às considerações finais.

\section{REFERENCIAL TEÓRICO}

Segundo Marinho (2007, p. 10), “o que define a moeda é a sua liquidez, isto é, a capacidade que ela possui de ser um ativo prontamente disponível e aceito para as mais diversas transações".

A moeda é um ativo financeiro utilizado na troca de bens e serviços no sistema econômico da sociedade. Ela desempenha algumas funções na economia de um país, sendo suas principais características: servir como meio ou instrumento de troca; funcionar como ativo financeiro que possui aquiescência; ser uma unidade de medida, permitindo contabilizar o valor dos produtos e serviços produzidos no mercado; também pode ser utilizada como reserva de valor, podendo ser guardada como forma de poupança para ser utilizada no futuro; e, finalmente, pode funcionar também como instrumento de especulação (CARVALHO et al., 2015; PASSOS; NOGAMI, 2015; SANT'ANA, 1997; VASCONCELLOS, 2011; MARINHO, 2007).

Ao longo da história da economia, a moeda (o dinheiro) esteve sempre relacionada com poder. No início, as moedas eram feitas de metais preciosos e, por isso, o valor estava intrínseco, por causa do valor do metal - fase conhecida como moeda metálica (MARINHO, 2007). Tempos depois, a moeda passou a ser impressa em papel, mas ainda seu valor estava relacionado à quantidade de ouro armazenada em algum cofre do banco central de determinado país (BARIVIERA et al., 2017). Finalmente, o papel moeda apresentou-se como o último estágio, com monopólio de produção do Estado e curso forçado, ainda sem o valor convertido em metal (MARINHO, 2007; SILVA; MONTEIRO, 2018).

A utilização da moeda foi o que viabilizou a implementação de um sistema de preço, ou seja, determinado bem ou serviço possui um valor e, desta forma, possibilitou-se a implementação de um sistema econômico baseado no dinheiro (MARTINS, 2016). 


\subsection{Moeda digital}

O dinheiro em sua forma eletrônica, através dos depósitos, está presente na vida de todos há mais de 50 anos. Atualmente, a moeda digital é um outro tipo de dinheiro eletrônico, cuja diferença com os depósitos está em que ela é um ativo que pode mudar de dono e o depósito eletrônico é uma reserva de valor que precisa de uma instituição intermediária para efetivar sua transação (DWYER, 2015).

Em meados da década de 1990, de acordo com Grinberg (2014), surgiram ideias sobre moedas totalmente virtuais, como a intitulada b-money, proposta por um grupo de hackers chamado Cyberpunks. Ainda segundo o mesmo autor, embora baseada em criptografia, esta moeda e outras contemporâneas possuíam como vulnerabilidade a sua própria característica virtual, possibilitando um gasto duplo. Justamente com o objetivo de contornar esta problemática o trabalho de Nakamoto (2008) foi publicado, com a advertência de que ainda hoje existem dúvidas sobre o verdadeiro autor (RUAS, 2017).

A moeda digital criptografada, em nível internacional a partir da criação do Bitcoin em 2008, procura dar resposta aos altos custos de transação monetária e aos problemas de segurança das moedas digitais, que dificultavam a sua legitimidade (BARIVIERA et al., 2017). Segundo Nakamoto (2008), essa moeda eletrônica engloba uma cadeia de assinaturas digitais, onde ocorrem transferências de valores através de códigos criptografados, ou seja, assinados digitalmente, o que dispensaria a dependência da confiança proporcionada por um sistema de controle da propriedade.

O sistema de funcionamento do Bitcoin é chamado de Peer-to-Peer, cujas transações e usuários são registrados na chamada Blockchain. Esse livro-caixa permite aos usuários de Bitcoin visualizar a contabilidade e as transações realizadas com a moeda. Essa tecnologia funciona por meio de um algoritmo criptografado, que a cada transação cria um registro das transferências mais recentes, conhecido como hash, um entrelaçado de números e letras, que protegem e garantem a segurança desse ativo, uma vez que se torna muito difícil para outros usuários ou pessoas manipularem uma transação (ARON, 2014; ULRICH, 2014).

\subsection{Regulamentação}

No mundo todo existem preocupações a respeito da regulamentação do Bitcoin. Alguns países, como o Equador, baniram a moeda, e os Estados Unidos criaram uma 
organização regulatória chamada de FinCEN, que opera individualmente nos Estados e regula as transações digitais (PIETERS; VIVANCO, 2017). A preocupação desses países é que as moedas digitais, como o Bitcoin, facilitariam o tráfico, a lavagem de dinheiro e a evasão de divisas. Por isso, muitos países ainda estão à procura de medidas jurídico-econômicas apropriadas para lidar com as operações virtuais e minimizar seus riscos, para, dessa forma, acompanhar as tendências e contribuir com a confiança do mercado (CAMPOS, 2015).

Um exemplo disso foi a classificação do Internal Revenue Service (órgão tributário) dos Estados Unidos, que declarou a moeda como propriedade (semelhante ao que fez o Canadá) e determinou que todas as empresas deveriam declarar todas as suas vendas feitas por meio deste ativo, assim como os lucros providos através da especulação (CAMPOS, 2015).

No Brasil, o Bitcoin está sendo comercializado e desperta cada vez mais o interesse dos investidores, mas o governo ainda não conseguiu se posicionar estrategicamente diante desse cenário. O Banco Central emitiu um comunicado recentemente (comunicado $\mathrm{n}^{\circ}$ 31.379, de 16/11/2017), alertando sobre os possíveis riscos, não só do Bitcoin, mas do mercado de criptomoedas em geral. Ressaltou que a moeda não é fiscalizada por qualquer entidade com autoridade monetária e não há garantias sobre esses ativos, deixando todo o risco na mão dos detentores (BRASIL, 2017).

Ainda, segundo Silva e Monteiro (2018), tem-se uma necessidade de melhor entendimento jurídico da tecnologia Blockchain e das moedas digitais no âmbito regulatório, uma vez que este marco não existe, o que é um problema potencial, já que não existe legislação própria para o assunto.

\section{MÉTODO}

Este trabalho, caracterizado como uma pesquisa bibliográfica e descritiva, buscou coletar e analisar, quantitativa e qualitativamente, estudos publicados em revistas científicas indexadas, com a expectativa de aumentar a familiaridade e mostrar a fronteira do conhecimento relacionado à primeira criptomoeda, o Bitcoin.

Assim, elaborou-se um protocolo de coleta com os seguintes critérios: Consideraramse as bases de dados Science Direct e Scielo; utilizou-se somente a palavra-chave de busca "Bitcoin"; o período considerado para a coleta das publicações foi entre janeiro de 2008 e 
abril de 2018; os idiomas das publicações incluídos na coleta dos trabalhos foram o inglês, português e espanhol.

No protocolo de coleta também foi considerado o critério relacionado aos tipos de estudos. Assim, na coleta incluíram-se: artigos de revisão; artigos completos de pesquisa; resumos de congressos; estudos de caso; relatórios de conferências; discussões ou debates científicos; editoriais; mini revisões e notas científicas - short communications. Por outro lado, excluíram-se os seguintes tipos de publicações: enciclopédias; capítulos de livro; revisões de livros; cartas; notícias; patentes; guias práticos; revisões de produtos; estudos de replicação; softwares; vídeo publicações.

\section{ANÁLISE E DISCUSSÃO DOS RESULTADOS}

A coleta de dados foi realizada em 5 de abril de 2018 e foram identificadas 432 publicações nas bases Science Direct e Scielo. Apesar de o Bitcoin ter surgido em 2008, é apenas a partir de 2011 que começam a ser publicados estudos científicos nas bases de dados consultadas. Entre 2011 e abril de 2018, observou-se um crescimento contínuo e rápido no número de publicações (Gráfico 1).

Gráfico 1 - Distribuição do número anual de publicações relacionadas com Bitcoin nas bases de dados Science Direct e Scielo, entre 2008 e abril de 2018.

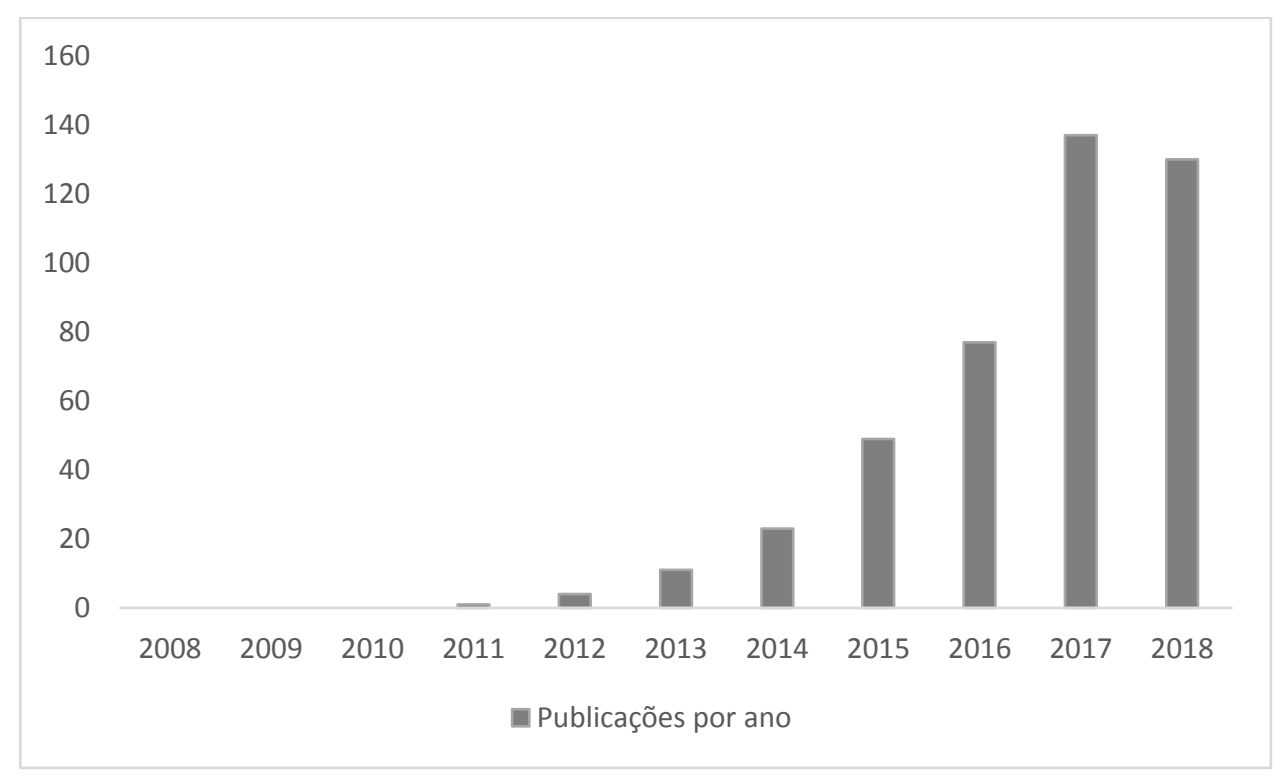

Fonte: Elaboração própria 
Na Tabela 1, mostra-se o número de publicações por tipo de trabalho, tipologia baseada na classificação fornecida na opção de busca avançada das bases de dados utilizadas, coletando-se 428 publicações na Science Direct e quatro na Scielo.

Observou-se que a maior quantidade de publicações é do tipo "artigo completo de pesquisa“, representando quase $82 \%$ do total. No caso das publicações tipo "estudo de caso", as bases de dados consultadas reportaram a existência de somente uma publicação dentre as 432. No entanto, após a análise dos títulos e resumos de cada um dos trabalhos, constatou-se que há pelo menos doze estudos de caso, equivalentes a 3\% do total. Acredita-se que a diferença entre o que foi identificado e o que foi caracterizado pelas próprias bases de dados deve-se ao equívoco dos autores dos trabalhos na hora de classificar a própria pesquisa no processo de submissão à revista científica.

Tabela 1 - Tipos de trabalhos científicos publicados sobre Bitcoin nas bases de dados Science Direct e Scielo, entre 2008 e abril de 2018.

\begin{tabular}{l|c}
\hline \multicolumn{1}{c|}{ Tipo de publicação } & Número de publicações \\
\hline Artigos de revisão & 21 \\
\hline Artigos completos de pesquisa & 353 \\
\hline Resumos em congresso & 2 \\
\hline Estudos de caso & 1 \\
\hline Relatórios de congresso & 0 \\
\hline Debates científicos & 6 \\
\hline Editoriais & 19 \\
\hline Mini revisões & 2 \\
\hline Notas científicas - Short communications & 28 \\
\hline TOTAL & 432 \\
\hline FoT &
\end{tabular}

Fonte: elaboração própria

O número de trabalhos que se caracterizam como artigos de revisão é baixo, menos de $5 \%$, devido à novidade do assunto, resultado que evidencia a importância desta pesquisa bibliográfica. Em relação ao idioma de publicação dos trabalhos, observando-se a manutenção da prática de divulgação científica de alto impacto, $99 \%$ das publicações foram escritas em inglês. Trabalhos publicados em português e espanhol foram encontrados uma única vez, para ambos os casos.

Foram analisadas também as palavras-chave de cada um dos artigos coletados, com a intenção de mostrar os assuntos mais abordados nos trabalhos relacionados com o Bitcoin. Identificaram-se 1751 palavras-chave, após a exclusão da palavra Bitcoin e dos seus sinônimos genéricos como: currency; criptomoney; digital currency; criptocurrency; alternative currency. 
Entre os assuntos que têm motivado maior número de pesquisas relacionadas ao Bitcoin (Tabela 2) está a chamada Blockchain, com aproximadamente 226 palavras-chave. A Blockchain é a base tecnológica e de segurança das operações do Bitcoin. Foram identificadas pesquisas sobre o nível de segurança e privacidade, identificaram-se ataques e potenciais ataques de hackers e discutiram-se formas de minimizar esses riscos. Ainda sobre o assunto abordado nas pesquisas coletadas, quase 100 palavras-chave são relacionadas com a oportunidade que o Bitcoin e a tecnologia Blockchain representam para o mercado ilegal ou Dark Market. Foram encontradas também pesquisas direcionadas à análise e busca de redução da comercialização de drogas lícitas e ilícitas, como cocaína, maconha, heroína, abortivos e drogas vendidas exclusivamente sob prescrição médica.

Podem-se ainda destacar as pesquisas relacionadas com o mercado financeiro, as questões políticas e o potencial de aplicação ou uso do Bitcoin e da Blockchain em outras áreas econômicas, tecnológicas e de conhecimento, como o setor de energia, a sustentabilidade, a economia solidária e a internet das coisas, entre outros assuntos.

Finalmente, outro assunto constantemente abordado é o relacionado com o mercado de Bitcoin, com quase 190 palavras-chave, onde se identificaram estudos sobre a avaliação de preços, possíveis bolhas, dinâmica das transações, eficiência e ineficiência do mercado.

Tabela 2 - Principais assuntos abordados pelas pesquisas encontradas.

\begin{tabular}{l|c|l}
\multicolumn{1}{c}{ Exemplo das principais palavras-chave } & $\begin{array}{l}\text { Quantidade } \\
\text { de palavras }\end{array}$ & \multicolumn{1}{c}{$\begin{array}{c}\text { Assunto geral } \\
\text { abordado }\end{array}$} \\
\hline $\begin{array}{l}\text { Confidentiality; Fraud; Data-protection; Hackers; } \\
\text { Ransomware; Security; Privacy; Attack; }\end{array}$ & 226 & $\begin{array}{l}\text { Bitcoin/Blockchain - } \\
\text { segurança }\end{array}$ \\
\hline $\begin{array}{l}\text { Anonymous; } \text { etc. } \\
\text { Bubbles; Cryptomarket; Efficiency; Exchange- } \\
\text { etc. }\end{array}$ & 186 arket; Online-payments; Prices; Volatility; & Mercado de Bitcoin \\
\hline $\begin{array}{l}\text { Dark; Drugs; Heroin; New-psychoactive- } \\
\text { substances; Black-market; Cocaine; Cannabis; } \text { etc. }\end{array}$ & 96 & $\begin{array}{l}\text { Bitcoin/Blockchain - } \\
\text { Dark Market }\end{array}$ \\
\hline $\begin{array}{l}\text { Bank; Crowdfunding; Crowdsourcing; Economic- } \\
\text { potential; Financial-innovation; Investment; }\end{array}$ & 80 & $\begin{array}{l}\text { Bitcoin - Mercado } \\
\text { financeiro }\end{array}$ \\
$\begin{array}{l}\text { Speculation; Sharing-economy; } \text { etc. } \\
\text { Disruptive-technologies; Innovation; IT; IoT; } \\
\text { Technology-evolution; Digital-innovation; } \text { etc. }\end{array}$ & 53 & $\begin{array}{l}\text { Bitcoin/Blockchain - } \\
\text { tecnologia disruptiva e } \\
\text { inovação }\end{array}$ \\
\hline $\begin{array}{l}\text { Carbon-emission; Climate-change; Ecology- } \\
\text { market; Electricity-system; Electricity-transaction; } \\
\text { Environment; Sustainability; Microgrid-control; } \\
\text { etc. }\end{array}$ & 50 & $\begin{array}{l}\text { Bitcoin/Blockchain - } \\
\text { energia e } \\
\text { sustentabilidade }\end{array}$ \\
\hline
\end{tabular}


(CONTINUAÇÃO)

\begin{tabular}{l|l|l}
\hline $\begin{array}{l}\text { Law; Institutionalization; Monetary-policy; } \\
\text { Political-economy; Regulation; Transforming- } \\
\text { government; } \text { etc. }\end{array}$ & 48 & $\begin{array}{l}\text { Bitcoin - políticas e } \\
\text { leis }\end{array}$ \\
$\begin{array}{l}\text { Crude-oil-market; Gold-market; Gold-price; etc. } \\
\begin{array}{l}\text { Outros temas: Deep-web; Algorithms; Altcoins; } \\
\text { Android; Artificial-intelligence; Big-data; Business- } \\
\text { models; Clinical-care; Copyright; Cyber-war; }\end{array}\end{array}$ & 18 & $\begin{array}{l}\text { Bitcoin/Blockchain - } \\
\text { outros mercados }\end{array}$ \\
$\begin{array}{l}\text { Distributed-systems; Internet; Ontology; etc. } \\
\text { Total }\end{array}$ & $\mathbf{1 7 5 1}$ & \\
\hline
\end{tabular}

Fonte: elaboração própria

Para uma melhor visualização, apresenta-se um gráfico de radar, mostrando a distribuição percentual dos principais temas dos artigos no total, excluindo-se a categoria outros temas. Percebe-se que as temáticas relacionadas à Blockchain e ao mercado de Bitcoin dominam os temas dos trabalhos científicos. Regulação e legislação, embora com relativa importância, não atingem 10\% dos temas totais (Gráfico 2).

Gráfico 2 - Distribuição dos principais temas dos artigos encontrados.

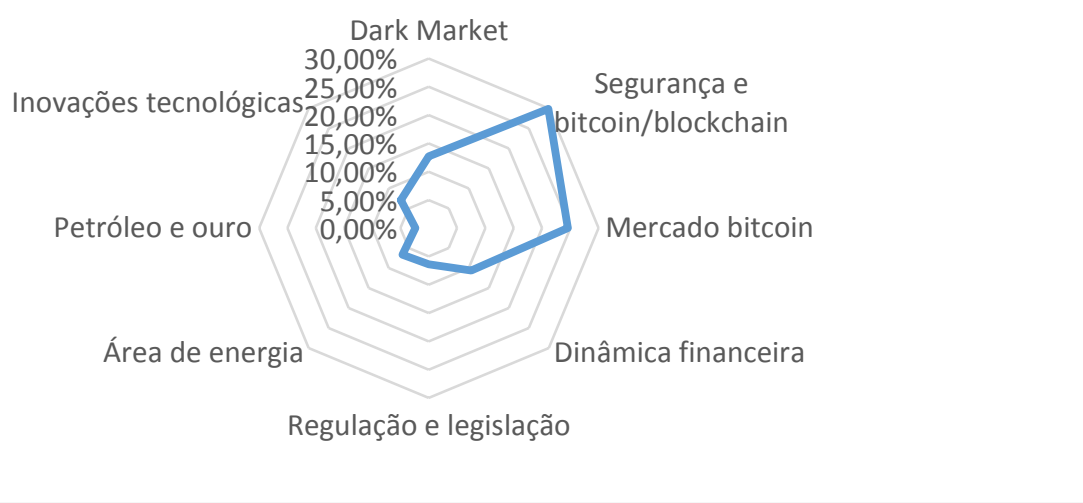

Fonte: elaboração própria

\subsection{Estado da arte sobre o conhecimento produzido acerca do Bitcoin e da Blockchain}

Visando apresentar a fronteira do conhecimento sobre Bitcoin como um resultado importante desta pesquisa, selecionaram-se as pesquisas de maior impacto científico, utilizando-se o critério de número de citações recebidas em artigos indexados em bases de dados científicas internacionais (Tabela 3 ). 
Tabela 3 - Artigos sobre Bitcoin com maior impacto na comunidade científica.

\begin{tabular}{|c|c|c|c|c|c|}
\hline & Título & Revista & Ano & $\begin{array}{c}\text { Tipo de } \\
\text { publicação }\end{array}$ & $\begin{array}{l}\text { Número } \\
\text { de } \\
\text { citações }\end{array}$ \\
\hline 1 & \begin{tabular}{|l|} 
The inefficiency of \\
Bitcoin
\end{tabular} & Economics Letters & 2016 & $\begin{array}{c}\text { Short } \\
\text { communication }\end{array}$ & 143 \\
\hline 2 & $\begin{array}{l}\text { Speculative bubbles in } \\
\text { Bitcoin markets? An } \\
\text { empirical investigation } \\
\text { into the fundamental } \\
\text { value of Bitcoin }\end{array}$ & Economics Letters & 2015 & $\begin{array}{c}\text { Short } \\
\text { communication }\end{array}$ & 132 \\
\hline 3 & $\begin{array}{l}\text { Bitcoin, gold and the } \\
\text { dollar - A GARCH } \\
\text { volatility analysis }\end{array}$ & $\begin{array}{l}\text { Finance } \quad \text { Research } \\
\text { Letters }\end{array}$ & 2016 & $\begin{array}{l}\text { Artigos } \\
\text { completos de } \\
\text { pesquisa }\end{array}$ & 121 \\
\hline 4 & $\begin{array}{l}\text { Designing microgrid } \\
\text { energy markets: A case } \\
\text { study: The Brooklyn } \\
\text { Microgrid }\end{array}$ & Applied Energy & 2018 & $\begin{array}{c}\text { Artigo completo } \\
\text { de pesquisa }\end{array}$ & 116 \\
\hline 5 & $\begin{array}{lr}\text { Putting the } & \text { sharing } \\
\text { economy } & \text { into } \\
\text { perspective } & \end{array}$ & $\begin{array}{l}\text { Environmental } \\
\text { Innovation and Societal } \\
\text { Transitions }\end{array}$ & 2017 & $\begin{array}{l}\text { Artigo completo } \\
\text { de pesquisa }\end{array}$ & 100 \\
\hline 6 & $\begin{array}{l}\text { IoT security: Review, } \\
\text { blockchain solutions, } \\
\text { and open challenges }\end{array}$ & $\begin{array}{l}\text { Future Generation } \\
\text { Computer Systems }\end{array}$ & 2018 & $\begin{array}{c}\text { Artigo completo } \\
\text { de pesquisa }\end{array}$ & 97 \\
\hline 7 & $\begin{array}{l}\text { Blockchain technology } \\
\text { in the chemical industry: } \\
\text { Machine-to-machine } \\
\text { electricity market }\end{array}$ & Applied Energy & 2017 & $\begin{array}{c}\text { Artigo completo } \\
\text { de pesquisa }\end{array}$ & 97 \\
\hline 8 & $\begin{array}{l}\text { The economics of } \\
\text { Bitcoin and similar } \\
\text { private digital currencies }\end{array}$ & $\begin{array}{l}\text { Journal of Financial } \\
\text { Stability }\end{array}$ & 2015 & $\begin{array}{c}\text { Artigo completo } \\
\text { de pesquisa }\end{array}$ & 96 \\
\hline 9 & $\begin{array}{l}\text { Hedging capabilities of } \\
\text { bitcoin. Is it the virtual } \\
\text { gold? }\end{array}$ & $\begin{array}{l}\text { Finance } \quad \text { Research } \\
\text { Letters }\end{array}$ & 2016 & $\begin{array}{c}\text { Artigo completo } \\
\text { de pesquisa }\end{array}$ & 93 \\
\hline 10 & $\begin{array}{l}\text { On the inefficiency of } \\
\text { Bitcoin }\end{array}$ & Economics Letters & 2017 & $\begin{array}{c}\text { Short } \\
\text { communication }\end{array}$ & 91 \\
\hline 11 & $\begin{array}{l}\text { On the hedge and safe } \\
\text { haven properties of } \\
\text { Bitcoin: Is it really more } \\
\text { than a diversifier? }\end{array}$ & Research & 2017 & $\begin{array}{c}\text { Artigo completo } \\
\text { de pesquisa }\end{array}$ & 91 \\
\hline 12 & $\begin{array}{l}\text { Price discovery of } \\
\text { cryptocurrencies: } \\
\text { Bitcoin and beyond }\end{array}$ & Economics Letters & 2018 & $\begin{array}{c}\text { Short } \\
\text { communication }\end{array}$ & 86 \\
\hline 13 & $\begin{array}{l}\text { 'Silk Road', the virtual } \\
\text { drug marketplace: A } \\
\text { single case study of user } \\
\text { experiences }\end{array}$ & $\begin{array}{l}\text { International Journal of } \\
\text { Drug Policy }\end{array}$ & 2013 & Debate & 86 \\
\hline
\end{tabular}


(CONTINUAÇÃO)

\begin{tabular}{|c|c|c|c|c|c|}
\hline 14 & $\begin{array}{l}\text { 'Surfing the Silk Road': } \\
\text { A study of users' } \\
\text { experiences }\end{array}$ & $\begin{array}{l}\text { International Journal of } \\
\text { Drug Policy }\end{array}$ & 2013 & $\begin{array}{l}\text { Artigo completo } \\
\text { de pesquisa }\end{array}$ & 83 \\
\hline 15 & 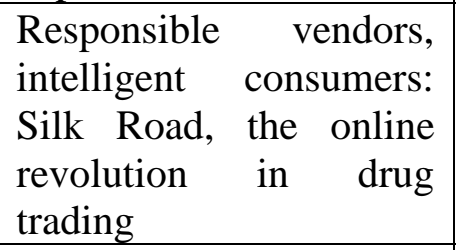 & $\begin{array}{l}\text { International Journal of } \\
\text { Drug Policy }\end{array}$ & 2014 & $\begin{array}{l}\text { Artigo completo } \\
\text { de pesquisa }\end{array}$ & 79 \\
\hline 16 & $\begin{array}{l}\text { Everything you always } \\
\text { wanted to know about } \\
\text { drug cryptomarkets* } \\
\text { (*but were afraid to ask) }\end{array}$ & $\begin{array}{l}\text { International Journal of } \\
\text { Drug Policy }\end{array}$ & 2016 & Editorial & 72 \\
\hline 17 & $\begin{array}{l}\text { Access control in the } \\
\text { Internet of Things: Big } \\
\text { challenges and new } \\
\text { opportunities }\end{array}$ & Computer Networks & 2017 & $\begin{array}{l}\text { Artigo de } \\
\text { Revisão }\end{array}$ & 72 \\
\hline 18 & $\begin{array}{l}\text { The inefficiency of } \\
\text { Bitcoin revisited: } \\
\text { dynamic approach }\end{array}$ & Economics Letters & 2017 & $\begin{array}{c}\text { Short } \\
\text { communication }\end{array}$ & 70 \\
\hline 19 & $\begin{array}{l}\text { A survey on the security } \\
\text { of blockchain systems }\end{array}$ & $\begin{array}{l}\text { Future Generation } \\
\text { Computer Systems }\end{array}$ & 2017 & $\begin{array}{c}\text { Artigo completo } \\
\text { de pesquisa }\end{array}$ & 69 \\
\hline 20 & $\begin{array}{l}\text { Some stylized facts of } \\
\text { the Bitcoin market }\end{array}$ & $\begin{array}{ll}\text { Physica A: } & \text { Statistical } \\
\text { Mechanics and its } \\
\text { Applications }\end{array}$ & 2017 & $\begin{array}{l}\text { Artigo completo } \\
\text { de pesquisa }\end{array}$ & 68 \\
\hline 21 & $\begin{array}{l}\text { Can volume predict } \\
\text { Bitcoin returns and } \\
\text { volatility? A quantiles- } \\
\text { based approach } \\
\end{array}$ & Economic Modelling & 2017 & $\begin{array}{l}\text { Artigo completo } \\
\text { de pesquisa }\end{array}$ & 65 \\
\hline 22 & $\begin{array}{l}\text { Exploring the dynamic } \\
\text { relationships between } \\
\text { cryptocurrencies and } \\
\text { other financial assets }\end{array}$ & Economics Letters & 2018 & $\begin{array}{c}\text { Short } \\
\text { communication }\end{array}$ & 64 \\
\hline 23 & $\begin{array}{l}\text { Hidden wholesale: The } \\
\text { drug diffusing capacity } \\
\text { of online drug } \\
\text { cryptomarkets } \\
\end{array}$ & $\begin{array}{l}\text { International Journal of } \\
\text { Drug Policy }\end{array}$ & 2016 & $\begin{array}{l}\text { Artigo completo } \\
\text { de pesquisa }\end{array}$ & 57 \\
\hline 24 & $\begin{array}{l}\text { The Internet-of-Things: } \\
\text { Review and research } \\
\text { directions }\end{array}$ & $\begin{array}{l}\text { International Journal of } \\
\text { Research in Marketing }\end{array}$ & 2017 & $\begin{array}{c}\text { Artigo completo } \\
\text { de pesquisa }\end{array}$ & 56 \\
\hline 25 & $\begin{array}{|ll|}\text { Price clustering in } \\
\text { Bitcoin }\end{array}$ & Economics Letters & 2017 & $\begin{array}{c}\text { Short } \\
\text { communication }\end{array}$ & 55 \\
\hline 26 & $\begin{array}{l}\text { Does Bitcoin hedge } \\
\text { global uncertainty? } \\
\text { Evidence from wavelet- } \\
\text { based quantile-in- } \\
\text { quantile regressions }\end{array}$ & $\begin{array}{l}\text { Finance } \\
\text { Letters }\end{array}$ & 2017 & $\begin{array}{l}\text { Artigo completo } \\
\text { de pesquisa }\end{array}$ & 55 \\
\hline
\end{tabular}


(CONTINUAÇÃO)

\begin{tabular}{|c|c|c|c|c|c|}
\hline 27 & $\begin{array}{l}1 \text { Blockchain's roles in } \\
\text { meeting key supply } \\
\text { chain management } \\
\text { objectives }\end{array}$ & $\begin{array}{l}\text { International Journal of } \\
\text { Information } \\
\text { Management }\end{array}$ & 2018 & $\begin{array}{c}\text { Artigo completo } \\
\text { de pesquisa }\end{array}$ & 53 \\
\hline 28 & $\begin{array}{l}\text { Bitcoin: Medium of } \\
\text { exchange or speculative } \\
\text { assets? }\end{array}$ & $\begin{array}{l}\text { Journal of International } \\
\text { Financial Markets, } \\
\text { Institutions and Money }\end{array}$ & 2017 & $\begin{array}{c}\text { Artigo completo } \\
\text { de pesquisa }\end{array}$ & 51 \\
\hline 29 & $\begin{array}{l}\text { Fentanyl, fentanyl } \\
\text { analogs and novel } \\
\text { synthetic opioids: A } \\
\text { comprehensive review } \\
\end{array}$ & Neuropharmacology & 2017 & $\begin{array}{l}\text { Artigo de } \\
\text { Revisão }\end{array}$ & 48 \\
\hline 30 & $\begin{array}{l}\text { The technology and } \\
\text { economic determinants } \\
\text { of cryptocurrency } \\
\text { exchange rates: The case } \\
\text { of Bitcoin }\end{array}$ & $\begin{array}{ll}\text { Decision } & \text { Support } \\
\text { Systems } & \end{array}$ & 2017 & $\begin{array}{c}\text { Artigo completo } \\
\text { de pesquisa }\end{array}$ & 45 \\
\hline 31 & \begin{tabular}{|lr} 
Safer & scoring? \\
Cryptomarkets, social \\
supply and drug market \\
violence
\end{tabular} & $\begin{array}{l}\text { International Journal of } \\
\text { Drug Policy }\end{array}$ & 2016 & $\begin{array}{c}\text { Artigo completo } \\
\text { de pesquisa }\end{array}$ & 44 \\
\hline 32 & $\begin{array}{l}\text { Big data: Dimensions, } \\
\text { evolution, impacts, and } \\
\text { challenges }\end{array}$ & Business Horizons & 2017 & $\begin{array}{c}\text { Artigo completo } \\
\text { de pesquisa }\end{array}$ & 44 \\
\hline 33 & $\begin{array}{l}\text { Blockchain's roles in } \\
\text { strengthening } \\
\begin{array}{l}\text { cybersecurity } \\
\text { protecting privacy }\end{array}\end{array}$ & $\begin{array}{l}\text { Telecommunications } \\
\text { Policy }\end{array}$ & 2017 & $\begin{array}{c}\text { Artigo completo } \\
\text { de pesquisa }\end{array}$ & 42 \\
\hline 34 & $\begin{array}{l}\text { Concepts of illicit drug } \\
\text { quality among darknet } \\
\text { market users: Purity, } \\
\text { embodied experience, } \\
\text { craft and chemical } \\
\text { knowledge }\end{array}$ & $\begin{array}{l}\text { International Journal of } \\
\text { Drug Policy }\end{array}$ & 2016 & $\begin{array}{c}\text { Artigo completo } \\
\text { de pesquisa }\end{array}$ & 41 \\
\hline 35 & $\begin{array}{l}\text { Informational efficiency } \\
\text { of } \quad \text { Bitcoin-An } \\
\text { extension }\end{array}$ & Computers \& Security & 2018 & $\begin{array}{c}\text { Short } \\
\text { communication }\end{array}$ & 41 \\
\hline
\end{tabular}

Fonte: elaboração própria

Foram selecionadas as 35 publicações com o maior número de citações em publicações indexadas. Juntas, essas publicações representam mais de $40 \%$ das 6410 citações que todas as pesquisas coletadas receberam desde que foram publicadas.

Em relação ao tipo de publicação, 23 dos artigos selecionados são publicações chamadas de artigo completo de pesquisa ou original article ou research article, que apresentam resultados inéditos de pesquisas científicas concluídas. Já as publicações do tipo short communication, oito entre os artigos selecionados, são uma forma de divulgação 
científica resumida de trabalhos, descobertas ou de resultados parciais que têm urgência de publicação, devido ao risco de ficarem ultrapassadas ou de outro pesquisador divulgar primeiro.

Sobre as revistas que publicam artigos de maior impacto relacionados com o Bitcoin, pode-se destacar a revista Economic Letters, com sete publicações que já foram citadas 641 vezes. A revista publica pesquisas na área de Economia, e em 2018 o seu fator de impacto foi 0,876. Outra revista que pode receber destaque é a International Journal of Drug Policy, também com sete publicações que já foram citadas 462 vezes. Essa revista publica pesquisas relacionadas ao uso de drogas e às políticas de drogas em contexto global, e em 2018 o seu fator de impacto foi 4,528. Uma última revista que pode ser destacada é a Finance Research Letters, que publicou quatro trabalhos que já foram citados 360 vezes. Essa revista publica pesquisas em todas as áreas das finanças, e em 2018 o seu fator de impacto foi 1,709.

Em relação ao período de publicação, pode-se mencionar que as pesquisas de maior impacto sobre o Bitcoin estão concentradas nos três últimos anos do período de coleta (2008 2018), indicando, junto ao já mencionado aumento no número de publicações, a crescente melhora na qualidade das pesquisas.

\subsubsection{O mercado de Bitcoin}

Sobre os principais assuntos que envolveram o Bitcoin, destacam-se as pesquisas que analisaram os fatores que influenciam a sua negociação no mercado. Urquhart (2016), no artigo "The inefficiency of Bitcoin", avaliou as informações históricas do preço da moeda e identificou que o seu mercado é ineficiente, mesmo que nos últimos anos tenha-se registrado uma melhora no cenário. Entretanto Nadarajah e Chu (2017), no artigo "On the inefficiency of Bitcoin”, refutaram os resultados obtidos por Urquhart (2016), mostrando que o mercado de Bitcoin tem potencial para ser eficiente. Por outro lado, Cheah e Fry (2015), no artigo "Speculative bubbles in Bitcoin markets? An empirical investigation into the fundamental value of Bitcoin", identificaram que o preço do Bitcoin tem um considerável componente especulativo, deixando-o mais suscetível às bolhas. Na mesma linha, Bariviera (2017), no artigo "The inefficiency of Bitcoin revisited: A dynamic approach" mostrou o processo da determinação e da volatilidade dos preços do Bitcoin. 
Ainda relacionado com o mercado de Bitcoin, ressaltam-se dois trabalhos. No artigo "A survey on the security of blockchain systems", Li et al. (2017) avaliaram sistematicamente os riscos na segurança do sistema Blockchain e propuseram alternativas para minimizar esses riscos. Kshetri (2017), no artigo "Blockchain's roles in strengthening cybersecurity and protecting privacy", comparou o sistema de segurança da nuvem e da Blockchain, indicando que o último oferece alto nível de proteção na privacidade dos usuários e, consequentemente, os setores público e privado deveriam investir mais para enriquecer o ecossistema Blockchain.

\subsubsection{O Bitcoin e a área financeira}

A área financeira também é um assunto de destaque nas principais publicações sobre Bitcoin. Dyhrberg (2016), no artigo "Bitcoin, gold and the dollar - A GARCH volatility analysis", avaliou a capacidade do Bitcoin como ativo financeiro, mostrando semelhanças com o ouro e o dólar em relação às capacidades de cobertura e de meio de troca, assim como meio de gerenciamento de risco. Bouri et al. (2017b) mostraram que é mais do que uma opção de diversificação de investimento, pois tem propriedades de opção estratégica de hedge, contribuindo com a minimização de risco para o investidor diante de oscilações de preço. Bouri et al. (2017a) reforçaram o papel do Bitcoin como opção estratégica de hedge contra as incertezas. Da mesma forma, Corbet et al. (2018), no artigo "Exploring the dynamics relationships between cryptocurrencies and other financial assets", mostraram que as moedas digitais, como o Bitcoin, podem oferecer benefícios no curto prazo para os investidores.

\subsubsection{O Bitcoin e o Dark Market}

Outra área que está chamando a atenção da ciência é o uso do Bitcoin e da tecnologia Blockchain no mercado de drogas. Hout e Bingham (2013a, 2013b, 2014) mostraram que o Bitcoin e a Blockchain oferecem na internet a infraestrutura necessária (anonimato, segurança, eficiência) para a compra e venda de drogas. Caracterizaram o perfil do principal comprador (homem, entre 18 e 25 anos, empregado, profissional formado e até com estudos de pós-graduação). Enquanto os vendedores, que se autodenominam como usuários 
responsáveis de drogas, decidem comercializar os produtos pela internet dadas as oportunidades de operar sob baixo risco, alta demanda, alta margem de lucro, segurança e anonimato. Por outro lado, Armenian et al. (2018) mostraram que o mercado de Fentanil, conhecido como o novo ópio sintético, vem constantemente crescendo. Já Albridge e DécaryHétu (2016) revelaram que as drogas tipo Ecstasy estão dominando o mercado no atacado.

\subsubsection{O Bitcoin e outras áreas de conhecimento}

Entre as publicações de maior impacto relacionadas com o Bitcoin, destacam-se pesquisas que relacionam a criptomoeda e a tecnologia Blockchain com outras áreas. Mengelkamp et al. (2018) mostraram o estado da arte das pesquisas que avaliam a comercialização de energia descentralizada utilizando a tecnologia Blockchain, pontuando as vantagens e a necessidade de regulação. Frenken e Schor (2017) avaliaram o Bitcoin desde a perspectiva do desenvolvimento da economia compartilhada, que poderá trazer vantagens ambientais e sociais para as atividades econômicas. Khan e Salah (2018) e Ng e Wakenshaw (2017) discutiram as pesquisas que relacionam as tecnologias Internet das Coisas (IoT) e Blockchain, ressaltando as potenciais vantagens para a segurança e a melhora da comercialização de produtos. Finalmente, pode-se destacar a pesquisa de Kshetri (2017), que avaliou as vantagens do uso da tecnologia Blockchain na gestão de cadeias de suprimento, melhorando o custo, qualidade, velocidade, confiabilidade, redução de riscos, sustentabilidade e a flexibilidade do processo.

Outro resultado identificado nesta pesquisa é relativo às bases de publicações científicas, como a Science Direct e a Scielo, que atualmente mostram variados tipos de indicadores para mensurar o impacto das pesquisas, como o número de leitores que acessaram a publicação, os downloads dos trabalhos, assim como a repercussão nas mídias sociais, como o número de compartilhamentos, comentários e tweets. A título de exemplo, selecionaram-se as dez publicações que tiveram o maior número de leitores (Tabela 4). 
Tabela 4 - Artigos relacionados ao Bitcoin com maior número de leitores nas bases Science Direct e Scielo

\begin{tabular}{l|c|c}
\hline \multicolumn{1}{c|}{ Título do artigo } & $\begin{array}{c}\text { Ano da } \\
\text { publicação }\end{array}$ & $\begin{array}{c}\text { Número de } \\
\text { leitores }\end{array}$ \\
\hline $\begin{array}{l}\text { IoT security: Review, blockchain solutions, and open } \\
\text { challenges }\end{array}$ & 2018 & 961 \\
\hline Putting the sharing economy into perspective & 2017 & 849 \\
\hline $\begin{array}{l}\text { 1 Blockchain's roles in meeting key supply chain } \\
\text { management objectives }\end{array}$ & 2018 & 672 \\
\hline A survey on the security of blockchain systems & 2017 & 651 \\
\hline $\begin{array}{l}\text { Blockchain in government: Benefits and implications of } \\
\text { distributed ledger technology for information sharing }\end{array}$ & 2017 & 634 \\
\hline $\begin{array}{l}\text { Designing microgrid energy markets: A case study: The } \\
\text { Brooklyn Microgrid }\end{array}$ & 2018 & 567 \\
\hline $\begin{array}{l}\text { Blockchain's roles in strengthening cybersecurity and } \\
\text { protecting privacy }\end{array}$ & 2017 & 564 \\
\hline $\begin{array}{l}\text { Big data: Dimensions, evolution, impacts, and challenges } \\
\text { Bhe Impacts of Robotics, Artificial Intelligence On }\end{array}$ & 2017 & 542 \\
\hline The Internet-of-Things: Review and research directions & 2015 & 528 \\
\hline Fon: & 2017 & 513 \\
\hline
\end{tabular}

Fonte: elaboração própria

Diferentemente dos primeiros resultados que mostraram o maior impacto científico das pesquisas relacionadas com as áreas mercadológicas, financeiras e do Dark Market, na Tabela 4 se pode perceber que os assuntos que mais chamaram a atenção dos leitores relacionam o Bitcoin e a Blockchain com outras áreas de conhecimento como: Internet das Coisas; Economia de Compartilhamento; Comercialização de Energia; Big Data; Robótica e Inteligência Artificial. Ao mesmo tempo, as pesquisas relacionadas com a segurança nas transações são o assunto de maior interesse dos leitores quando o Bitcoin e a Blockchain são o foco único na busca.

Pode-se ressaltar que os indicadores que avaliam o interesse despertado pelos trabalhos publicados, como o número de leitores, potencialmente sinalizarão os assuntos que futuramente ganharão destaque no número de citações em novos trabalhos de pesquisa.

\section{CONSIDERAÇÕES FINAIS}

Esta pesquisa bibliográfica mostrou que o número de publicações científicas em relação ao Bitcoin tem aumentado rapidamente. Uma evidência desse fato é que em 2018 o número de trabalhos publicados até o começo de abril, quando foi feita a coleta das publicações, quase alcançou as divulgações científicas indexadas em todo o ano de 2017. 
O amplo e diverso potencial de uso e aplicação do Bitcoin e da tecnologia Blockchain está incrementando o interesse das diversas áreas da ciência, como economia, finanças, energia, sustentabilidade, saúde, IoT, Big Data, entre outros.

Por outro lado, quando o assunto é a criptomoeda e o criptomercado, identificam-se pesquisas que buscam entender o seu funcionamento, as vantagens e riscos para os usuários, assim como as oportunidades que o chamado "mercado negro" tem para se desenvolver através de uma nova infraestrutura que a internet oferece, como o Bitcoin e a Blockchain, que garante rapidez, segurança e privacidade.

Assim, entende-se que a pesquisa científica em relação ao Bitcoin e à Blockchain e suas diversas aplicações deverá continuar em ascensão, identificando novas oportunidades e riscos, propondo alternativas para melhorar a eficiência do mercado e das moedas digitais, assim como para aproveitar a interação com outras áreas de conhecimento. As moedas digitais e a Blockchain podem ser retratadas como uma inovação tecnológica disruptiva de impacto global, e a ciência deverá continuar contribuindo para a sua consolidação e constante desenvolvimento.

\section{REFERÊNCIAS}

ALBRIDGE, J.; DÉCARY-HÉTU, D. Hidden wholesale: The drug diffusing capacity of online drug cryptomarkets. International Journal of Drug Policy, v. 35, p. 7-15.2016. <doi.org/10.1016/j.drugpo.2016.04.020>

ARMENIAN, P.; VO, K. T.; BARR-WALKER, J.; LYNCH, K. L. Fentanyl, fentanyl analogs and novel synthetic opioids: A comprehensive review. Neuropharmacology, v. 134, p. 121132, 2018. <doi.org/10.1016/j.neuropharm.2017.10.016>

ARON, J. Building on Bitcoin. New Scientist, p. 19-20, 8 fev. 2014. Disponível em: $<$ https://www.newscientist.com/article/mg22129553-700-bitcoin-how-its-core-technologywill-change-the-world/>.

BALCILAR, M.; BOURI, E.; GUPTA, R.; ROUBAUD, D. Can volume predict Bitcoin returns and volatility? A quantiles-based approach. Economic Modelling, v. 64, p. 74-81, 2017. <https://doi.org/10.1016/j.econmod.2017.03.019>

BARIVIERA, A. F. The inefficiency of Bitcoin revisited: A dynamic approach. Economics Letters, v. 161, p. 1-4, 2017. <doi.org/10.1016/j.econlet.2017.09.013> 
BARIVIERA, A. F. et al. Some stylized facts of the Bitcoin market. Physica A: Statistical Mechanics and its Applications, v. 484, p. 82-90, 2017. <doi.org/10.1016/j.physa.2017.04.159>.

BAUR, D. G.; HONG, K.; LEE, A. D. Bitcoin: Medium of exchange or speculative assets? Journal of International Financial Markets, Institutions and Money, v. 54, p. 177-189, 2018. <doi.org/10.1016/j.intfin.2017.12.004>.

BOURI, E.; GUPTA, R.; TIWARI, A. K.; ROUBAUD, D. Does Bitcoin hedge global uncertainty? Evidence from wavelet-based quantile-in-quantile regressions. Finance Research Letters, v. 23, p. 87-95, 2017a. <doi.org/10.1016/j.frl.2017.02.009>

BOURI, E.; MOLNÁR, P.; AZZI, G.; ROUBAUD, D.; HAGFORS, L. I. On the hedge and safe haven properties of Bitcoin: Is it really more than a diversifier? Finance Research Letters, v. 20, p. 192-198, 2017b. <doi.org/10.1016/j.frl.2016.09.025>

BRASIL. Banco Central. Comunicado no 31.379, de 16 de nov. 2017. Alerta sobre os riscos decorrentes de operações de guarda e negociação das denominadas moedas virtuais. Disponível em: <http://www.bcb.gov.br/pre/normativos/busca/normativo.asp?numero=31379\&tipo=Comunic ado\&data=16/11/2017>. Acesso em: 02 dez. 2017.

CALVETTE, R. C. BITCOIN: um estudo sobre a moeda digital e deflação. 2015. [26] f., il. Monografia (Bacharelado em Administração) - Universidade de Brasília, Brasília, 2015. Disponível em: <http://bdm.unb.br/bitstream/10483/12438/1/2015_RafaelCintraCalvette.pdf>. Acesso em: 30 ago. 2017.

CAMPOS, G. I. R. V. Bitcoin: consequências jurídicas do desenvolvimento da moeda virtual. Revista Brasileira de Direito, Passo Fundo, v. 11, n. 2, p. 77-84, dez. 2015. ISSN 22380604. Disponível em: <https://seer.imed.edu.br/index.php/revistadedireito/article/view/769>.

CARVAlHO, F. J. C. de.; SOUZA, F. E. P. de.; SICSÚ, J.,; De PAULA, L. F. R.; STUDART, R. Economia Monetária e financeira: teoria e política. 3. Ed. Rio de Janeiro: Elsevier, 2015.

CHEAH, E.-T.; FRY, J. Speculative bubbles in Bitcoin markets? An empirical investigation into the fundamental value of Bitcoin. Economics Letters, v. 130, p. 32-36, 2015. <doi.org/10.1016/j.econlet.2015.02.029>

CORBET, S.; MEEGAN, A.; LARKIN, CH.; LUCEY, B.; YAROVAYA, L. Exploring the dynamic relationships between cryptocurrencies and other financial assets. Economics Letters, v. 165, p. 28-34, 2018. <doi.org/10.1016/j.econlet.2018.01.004>

DWYER, G. P. The economics of Bitcoin and similar private digital currencies. Journal of Financial Stability, v. 17, p. 81-91, 2015. <doi.org/10.1016/j.jfs.2014.11.006>

DYHRBERG, A. H. Bitcoin, gold and the dollar - a GARCH volatility analysis. Finance Research Letters, v. 16, p. 85-92, 2016. <doi.org/10.1016/j.frl.2015.10.008> 
EYAL, I.; SIRER, E. G. Majority Is Not Enough: Bitcoin Mining Is Vulnerable. Lecture Notes in Computer Science, vol. 8437. Springer, Berlin, Heidelberg 2014. <doi.org/10.1007/978-3-662-45472-5_28>

FRENKEN, K.; SCHOR, J. Putting the sharing economy into perspective. Environmental

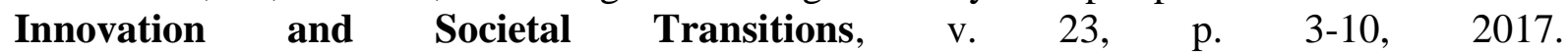
<doi.org/10.1016/j.eist.2017.01.003>

GRINBERG, Reuben. Bitcoin: An innovative alternative digital currency. Hastings Sci. Tech. LJ, v. 4, p. 159, 2014. Disponível em: <https://papers.ssrn.com/sol3/papers.cfm?abstract_id=1817857>. Acesso em: 07/10/2019.

HOUT, M. C. V.; BINGHAM, T. Responsible vendors, intelligent consumers: silk road, the online revolution in drug trading. International Journal of Drug Policy, v. 25, p. 183-189, 2014. <doi.org/10.1016/j.drugpo.2013.10.009>

HOUT, M. C. V.; BINGHAM, T. 'Silk Road', the virtual drug marketplace: A single case study of user experiences. International Journal of Drug Policy, v. 24, p. 385-391, 2013a. <doi.org/10.1016/j.drugpo.2013.01.005>

HOUT, M. C. V.; BINGHAM, T. 'Surfing the Silk Road': A study of users' experiences. International Journal of Drug Policy, v. 24, p. 524-529, $2013 \mathrm{~b}$. <doi.org/10.1016/j.drugpo.2013.08.011>

KHAN, M. A.; SALAH, K. IoT security: Review, blockchain solutions, and open challenges. Future Generation Computer Systems, v. 82, p. 395-411, 2018. $<$ doi.org/10.1016/j.future.2017.11.022>

KSHETRI, N. Blockchains's roles in strengthening cybersecurity and protecting privacy. $\begin{array}{llllllll}\text { Telecommunications } & \text { Policy, } & \text { v. } 41, & \text { i. } 10, & \text { p. } & 1027-1038, & \end{array}$ <doi.org/10.1016/j.telpol.2017.09.003>

LI, X.; JIANG, P.; CHEN, T.; LUO, X.; WEN, Q. A survey on the security of blockchain $\begin{array}{llll}\text { systems. Future Generation Computer } & \text { Systems, } & \end{array}$ <doi.org/10.1016/j.future.2017.08.020>

MARTINS, A. N. da G. L. Quem tem medo do bitcoin? o funcionamento das moedas criptografadas e algumas perspectivas de inovações institucionais. Revista Jurídica Luso Brasileira, Rio de Janeiro, ano 2, n. 3, p. 137-171, 2016. Disponível em: <http://www.cidp.pt/publicacoes/revistas/rjlb/2016/3/2016_03_0137_0171.pdf>

MARINHO, H. Economia Monetária: Teorias e a experiência brasileira. Rio de Janeiro: Editora Ciência Moderna Ltda., 2007.

MENGELKAMP, E.; GARTTNER, J.; ROCK, KERSTIN; KESSLER, S.; WEINHARDT, $\mathrm{CH}$. Designing microgrid energy markets: A case study: The Brooklyn Microgrid. Applied Energy, v. 210, p. 870-880, 2018. <doi.org/10.1016/j.apenergy.2017.06.054>

NADARAJAH, S.; CHU, J. On the inefficiency of Bitcoin. Economics Letters, v. 150, p. 69, 2017. <doi.org/10.1016/j.econlet.2016.10.033> 
NAKAMOTO, Satoshi. Bitcoin: A Peer-to-Peer Electronic Cash System. 2008. Disponível em: <http://bitcoin.org/bitcoin.pdf>. Acesso em: 30 ago. 2017.

NEW SCIENTIST. Bitcoin: bringer of the The b-commerce revolution. New Scientist, p. 3, 30 nov. 2013. Disponível em: <https://doi.org/10.1016/S0262-4079(13)62759-1〉.

NG, I. C. L.; WAKENSHAW, S. Y. L. The Internet-of-Things: Review and research directions. International Journal of Research in Marketing, v. 34, p. 3-21, 2017. <doi.org/10.1016/j.ijresmar.2016.11.003>

PASSOS, C. R. M; NOGAMI, O. Princípios de economia. 6. ed. São Paulo, SP: Cengage Learning, 2015.

PIETERS, G.; VIVANCO, S. Financial Regulations and Price Inconsistencies across Bitcoin Markets. Information Economics and Policy, v. 39, p. 1-14, 2017. <doi.org/10.1016/j.infoecopol.2017.02.002>

PINTO, T. C. Aspectos inovativos do bitcoin, microestrutura de mercado e volatilidade de preços. Dissertação de Mestrado em Economia - Universidade Federal da Paraíba, João Pessoa, 2015. Disponível em: <https://repositorio.ufpb.br/jspui/handle/tede/8321>. Acesso em: 5 dez. 2017.

PINZÓN, C.; ROCHA, C. Double-spend Attack Models with Time Advantange for Bitcoin. Electronic Notes in Theoretical Computer Science, v. 329, p. 79-103, 2016. <doi.org/10.1016/j.entcs.2016.12.006>

RUAS, B. BITCOIN: Uma aplicação dos modelos de séries temporais autorregressivos. 2017. Trabalho de Conclusão de Curso (Bacharelado) - Escola Superior de Ciências Sociais, Universidade Estadual do Amazonas, Amazonas, 2017.

SANT'ANA, J. A. Economia monetária: a moeda em uma economia globalizada. Brasília, DF: Editora da UnB, 1997.

SILVA, S. C., MONTEIRO, V. B. A Sistemática Brasileira de Segurança da Atividade Financeira no ciberespaço e a atual (in) aplicabilidade às Initial Coin Offerings (Icos) e aos seis atios virtuais. Contextus - Revista Contemporânea de Economia e Gestão, v. 16, p. 6283, 2018. Disponível em: <http://www.periodicos.ufc.br/contextus/article/view/33320>. Acesso em: 07/10/2019.

SILVEIRA, F. A. Bitcoin como ativo em carteiras de investimentos: uma análise com base no modelo de portfólio de Markowitz. Universidade do Extremo Sul Catarinense (UNESC), 2015. Disponível em: <http://repositorio.unesc.net/handle/1/3525>. Acesso em: 30 ago. 2017.

ULRICH, F. Bitcoin: A Moeda na Era Digital. São Paulo: Instituto Ludwig von Mises Brasil, 2014. Disponível em: <http://www.informatrader.com.br/datafiles/conteudo_downloads/9/Fernando-UlrichBitcoin.pdf>. Acesso em: 30 ago. 2017. 
URQUHART, A. The inefficiency of Bitcoin. Economics Letters. v. 148, p. 80-82, 2016. $<$ doi.org/10.1016/j.econlet.2016.09.019>

VASCONCELLOS, M. A. S. de. Economia micro e macro: teoria e exercícios, glossário com os 300 principais conceitos econômicos. 5. ed. São Paulo, SP: Atlas, 2011. 